\title{
Avaliação do efeito do agente compatibilizante (PP-g-MA) em misturas PP/Amido termoplástico
}

\section{Evaluation of the effect of compatibilizing agent (PP-g-MA) on PP/Thermoplastic starch blends}

Carlos Ivan Ribeiro de Oliveira ${ }^{1}$, Vinícius Pereira de Almeida ${ }^{1}$, Marisa Cristina Guimarães Rocha ${ }^{1}$, Joaquim Teixeira de Assis ${ }^{1}$

\author{
${ }^{1}$ Instituto Politécnico, Universidade do Estado do Rio de Janeiro, R. Bonfim 25, Bloco 1, 28625-579, Nova Friburgo, Rio \\ de Janeiro, Brasil. \\ e-mail: carlosivanr@gmail.com, almeidapvinicius@gmail.com,mrocha@iprj.uerj.br, joaquim@iprj.uerj.br
}

\section{RESUMO}

O crescente desperdício de materiais plásticos no meio ambiente juntamente com a preocupação com a futura escassez do petróleo tem aumentado o interesse por polímeros biodegradáveis, como o amido. Nos últimos anos, o amido tem sido utilizado em misturas com diferentes polímeros sintéticos. Essas misturas têm sido compatibilizadas por meio do uso de agentes compatibilizantes do tipo copolímeros. O presente trabalho tem como objetivo avaliar a influência da adição do copolímero graftizado (PP-g-MA) nas propriedades mecânicas, térmicas e morfológicas de misturas de polipropileno (PP) e amido termoplástico (TPS). O TPS foi obtido em uma extrusora monorosca com $20 \%(\mathrm{~m} / \mathrm{m})$ de glicerol, usado como plastificante. Posteriormente, misturas PP/TPS $80: 20 \%(\mathrm{~m} / \mathrm{m})$ foram processadas em uma extrusora de rosca dupla co-rotacional, na presença de 3,5 e $7 \%(\mathrm{~m} / \mathrm{m})$ do PP-g-MA. A morfologia das misturas foi avaliada através de microscopia eletrônica de varredura. Os resultados mostraram uma redução significativa da fase dispersa de amido, com a adição do compatibilizante. As misturas processadas em presença do PP-g-MA apresentaram uma distribuição mais homogênea da fase de amido na matriz de PP. Os resultados dos ensaios de resistência à tração mostraram que o módulo do PP sofre um ligeiro aumento, com a adição do amido e do compatibilizante. A resistência das misturas no ponto de escoamento apresentou valores similares aos do PP. Apesar disso, a deformação na ruptura diminui drasticamente com a incorporação do amido. A adição de amido ao polipropileno, entretanto, aumenta significativamente a resistência ao impacto do polímero sintético. Com a adição de PP-g-MA à mistura PP/TPS, a resistência ao impacto sofre uma queda, que pode ser atribuída a redução da fase de amido na mistura. A estabilidade térmica das misturas PP/TPS sofre um ligeiro aumento com a incorporação do agente compatibilizante. Os materiais obtidos podem ser utilizados em substituição ao PP.

Palavras-chave: Polipropileno, Amido termoplástico, Misturas, Morfologia, Agente compatibilizante.

\begin{abstract}
The growing waste of plastics in the environment coupled with concerns about future oil shortages has increased interest in biodegradable polymers such as starch. In recent years, starch has been used in blends with different synthetic polymers. Such blends have been compatibilized by the use of compatibilizing agents of the copolymer type. In this study, the morphological, mechanical and thermal properties of polypropylene (PP)/thermoplastic starch (TPS) blends obtained in the presence of the compatibilizing agent (PP-g-MA) were evaluated. The thermoplastic starch was obtained in a single screw extruder with $20 \%$ (mass/mass) of glycerol used as plasticizer. PP/TPS blends were prepared by melt mixing in a co-rotating twin-screw extruder. The concentration of the compatibilizing agent was 3,5 and 7\% (mass/mass). The morphology of the blends was evaluated by scanning electron microscopy. The results showed a significant reduction of the dispersed starch phase with the addition of the PP-g-MA. The blends obtained in the presence of PP-g-MA showed a more homogeneous distribution of the starch phase in the PP matrix. The results obtained with the
\end{abstract}


tensile tests showed that the elastic modulus of PP undergoes a slight increase with the addition of the starch and the compatibilizer. The tensile strength of the PP was not significantly affected. However, there was a significant decrease of the elongation at break with the incorporation of starch. The starch incorporated in the PP matrix led to a significant increase in the impact strength of the final material. However, with the addition of PP-g-MA to the PP/TPS blend, the impact strength falls. This result can be attributed to the reduction of the starch phase in the PP matrix. The thermal stability of the PP/TPS blends was slightly increased with the incorporation of the compatibilizing agent. The materials obtained can be used instead of PP.

Keywords: Polypropylene, Thermoplastic starch, Blends, Morphology, Compatibilizing agent.

\section{INTRODUÇÃO}

Nos últimos anos, o desenvolvimento crescente da indústria de polímeros tem promovido um descarte cada vez maior de polímeros sintéticos no meio ambiente. Essa quantidade crescente de lixo plástico, juntamente com a preocupação com a futura escassez do petróleo tem aumentado o interesse por materiais poliméricos biodegradáveis, como o amido [1]. A aplicação desses materiais na área de embalagens, no campo da medicina e em setores automotivos em substituição aos polímeros sintéticos convencionais tem sido explorada por muitos centros de pesquisa e indústrias [2].

O amido é o principal polissacarídeo de reserva de carboidratos do reino vegetal, e é acumulado por plantas verdes sob a forma de grânulos, sendo composto por duas principais macromoléculas: amilose, que é essencialmente linear e amilopectina, que apresenta diversas ramificações ao longo de sua cadeia [3,4]. As vantagens do uso de amido em substituição aos polímeros sintéticos, é que esse bipolímero pode ser encontrado abundantemente na natureza, apresenta um baixo custo, pode ser misturado com diferentes polímeros sintéticos, pode ser utilizado como carga para a diminuição do custo do produto final, além de ser totalmente biodegradável [4]. As desvantagens de materiais baseados em amido termoplástico (TPS), material obtido após plastificação do amido in natura na presença de glicerol, em extrusora ou em misturadores [3], é que o TPS é um material hidrofílico, que absorve facilmente a umidade do ambiente. A absorção de umidade pelo amido termoplástico leva a uma redução significativa em suas propriedades mecânicas [5,6].

A mistura de TPS com polímeros sintéticos convencionais tem sido uma das alternativas mais utilizadas nos últimos anos para superar as desvantagens da utilização do amido na indústria de polímeros [7-9]. No entanto, o desenvolvimento dessas misturas, na maioria das vezes, leva a obtenção de materiais com baixas propriedades mecânicas. Isso ocorre devido à baixa miscibilidade entre o amido e a maioria dos polímeros sintéticos convencionais, já que o amido é um polímero hidrofílico, enquanto a maioria dos polímeros sintéticos convencionais apresenta um forte caráter hidrofóbico.

Diversos trabalhos têm avaliado a compatibilização de misturas poliméricas de amido com polímeros sintéticos por meio da utilização de agentes compatibilizantes comerciais, do tipo copolímeros [3,10-12]. Geralmente, os copolímeros utilizados são copolímeros em bloco e/ou copolímeros graftizados. Esses compatibilizantes (também chamados de modificadores interfaciais ou emulsificantes) são espécies macromoleculares que atuam na interface de misturas poliméricas imiscíveis. Esses agentes compatibilizantes promovem a redução da tensão interfacial, além de proporcionar uma melhor dispersão de uma fase polimérica em outra [13].

O polipropileno (PP) é um dos polímeros apolares mais utilizados na indústria nos últimos anos, principalmente devido ao seu baixo custo, baixa densidade, boas propriedades térmicas e mecânicas e excelente processabilidade. Diferentemente de misturas de polietileno e amido termoplástico, há na literatura muito poucos trabalhos sobre misturas de polipropileno e amido [7].

O objetivo do presente trabalho é avaliar a compatibilização de misturas de polipropileno e amido termoplástico, em presença do agente compatibilizante polipropileno graftizado com anidrido maleico (PP-gMA). As misturas foram obtidas em extrusora de rosca dupla co-rotacional e avaliadas quanto a morfologia, propriedades mecânicas e propriedades térmicas. O teor de amido presente nas misturas foi de $20 \%(\mathrm{~m} / \mathrm{m})$. O teor de PP-g-MA adicionado às misturas foi de 3, 5 e $7 \%(\mathrm{~m} / \mathrm{m})$. Os diferentes materiais obtidos foram caracterizados através de microscopia eletrônica de varredura (MEV), ensaios de resistência à tração, ensaios de resistência ao impacto e análise termogravimétrica (TGA).

\section{MATERIAIS E MÉTODOS}

\subsection{Materiais}

O polipropileno utilizado no presente trabalho foi o homopolímero PP H501HC doado pela Braskem. Segun- 
do o fornecedor, esse homopolímero apresenta um índice de fluidez de $3,5 \mathrm{~g} / 10 \mathrm{~min}\left(230^{\circ} \mathrm{C} / 2,16 \mathrm{~kg}\right)$. O amido de milho (Amidex 3001) foi doado pela Ingredion Brasil Ingredientes Industriais Ltda. (Mogi Guaçu, SP). O glicerol P.A., usado como plastificante, foi fornecido pela Sigma-Aldrich (Duque de Caxias, RJ). O polipropileno graftizado com anidrido maleico (PP-g-MA), Polybond 3200, com índice de fluidez de 109 g/10 $\min \left(190^{\circ} \mathrm{C} / 2,16 \mathrm{~kg}\right)$, foi fornecido pela Chemtura Indústria Química do Brasil Ltda.

\subsection{Obtenção das pré-misturas de amido de milho}

Pré-misturas de amido in natura e glicerol foram homogeneizadas em um béquer de plástico, sob agitação mecânica a $450 \mathrm{rpm}$, por um período de 10 minutos em um misturador convencional IKA, modelo RW20, de forma a favorecer a incorporação do plastificante ao amido. $\mathrm{O}$ teor de glicerol adicionado às amostras de amido foi de $20 \% \mathrm{~m} / \mathrm{m}$ (baseado no peso seco do amido). As pré-misturas foram condicionadas em sacos plásticos fechados para evitar a perda de material, e mantidas em refrigerador por 48 horas, a fim de permitir a completa absorção do plastificante pelo amido.

\subsection{Obtenção do amido termoplástico (TPS)}

O amido termoplástico (TPS) foi obtido a partir das pré-misturas de amido de milho, em uma extrusora monorosca AX Plásticos, modelo AX30:32, com um perfil de temperatura de 70/90/90/90 ${ }^{\circ} \mathrm{C}$ e velocidade de rotação de $90 \mathrm{rpm}$. $\mathrm{O}$ amido termoplástico foi resfriado ao ar livre sob temperatura média de $23^{\circ} \mathrm{C}$ e umidade relativa do ar em torno de $50 \%$ durante 2 horas e, posteriormente, granulado em máquina peletizadora.

\subsection{Preparação das misturas PP/PP-g-MA/TPS}

A amostra de polipropileno foi previamente seca em estufa a $60^{\circ} \mathrm{C}$ durante 24 horas. $\mathrm{O}$ agente compatibilizante (PP-g-MA), por ser um material muito reativo, foi apenas mantido em dessecador, durante o mesmo período. Após secagem, os materiais foram misturados manualmente em concentrações pré-determinadas (Tabela 1). O PP puro e as diferentes misturas foram processados em extrusora de rosca dupla co-rotacional Leistritz modelo ZSE 18 MAXX, com perfil de temperatura das diferentes zonas de aquecimento da extrusora de 160/170/170/180/180/185/185/190/190/190 ${ }^{\circ} \mathrm{C}$, da zona de alimentação até a matriz da extrusora, respectivamente. A velocidade de rotação das roscas durante o processamento foi mantida em $500 \mathrm{rpm}$. A dosagem do material na zona de alimentação foi de $5 \mathrm{~kg} / \mathrm{h}$. As condições de processamento das diferentes misturas foram previamente definidas por meio de testes preliminares efetuados com amostras de polipropileno virgem. Os materiais extrusados foram resfriados em água fria e, posteriormente, granulados em máquina peletizadora para posterior caracterização e obtenção de corpos de prova para realização de ensaios mecânicos.

Tabela 1: Composição das misturas PP/PP-g-MA/TPS.

\begin{tabular}{l|l|l|l}
\hline COMPOSIÇÃO & \multicolumn{3}{|c}{ PROPORÇÃO DOS COMPONENTES (\%) } \\
\hline & PP & TPS & PP-g-MA \\
\hline PP & 100 & 0 & 0 \\
\hline PP/TPS 80/20\% (m/m) & 80 & 20 & 0 \\
\hline PP/PP-g-MA/TPS 77/3/20\% (m/m) & 77 & 20 & 3 \\
\hline PP/PP-g-MA/TPS 75/5/20\% (m/m) & 75 & 20 & 5 \\
\hline PP/PP-g-MA/TPS 73/7/20\% $(\mathrm{m} / \mathrm{m})$ & 73 & 20 & 7 \\
\hline
\end{tabular}

\section{5 Índice de fluidez (MFI)}

$\mathrm{O}$ índice de fluidez do polipropileno e das diferentes misturas foi determinado em um plastômetro de extrusão CEAST 7021. As medidas de MFI foram determinadas a $230^{\circ} \mathrm{C}$, sob carga de $2,16 \mathrm{~kg}$ e tempo de corte de 30 segundos, de acordo com a norma ASTM D1238 [14]. O valor de índice de fluidez (MFI) foi calculado através da média aritmética de dez amostras para cada experimento.

\subsection{Obtenção de corpos de prova para ensaios mecânicos}

Corpos de prova para a realização de ensaios de resistência à tração e resistência ao impacto foram obtidos através de moldagem por injeção, efetuado em injetora Arburg, modelo Allrounder 270 S (400-170) com perfil de temperatura das diferentes zonas de aquecimento da injetora de $200 / 190 / 180 / 170 / 160^{\circ} \mathrm{C}$. A pressão 
de injeção usada foi de 800 bar e a velocidade de injeção de $30 \mathrm{~cm}^{3} / \mathrm{s}$. O tempo de resfriamento foi de 30 segundos. Para os ensaios de resistência a tração, corpos de prova tipo I foram obtidos, de acordo com a norma ASTM D638 [15]. Corpos de prova para ensaios de resistência ao impacto foram obtidos, de acordo com a norma ASTM D256 [16].

\subsection{Avaliação das propriedades mecânicas de tração}

Ensaios de resistência à tração das diferentes amostras foram realizados em Máquina Universal de Ensaios SHIMADZU, modelo AG-X Plus, equipada com garras para tração com distância de $50 \mathrm{~mm}$ e célula de carga de $5 \mathrm{kN}$. A velocidade de afastamento das garras foi de $20 \mathrm{~mm} / \mathrm{min}$. Os ensaios foram realizados de acordo com a norma ASTM D638 [15], com corpos de prova do tipo I. No mínimo, 7 corpos de prova para cada amostra foram usados para a obtenção dos dados mecânicos. As amostras foram analisadas após condicionamento a uma temperatura média de $21^{\circ} \mathrm{C}$ e umidade relativa do ar em torno de $50 \%$ durante 48 horas.

\subsection{Resistência ao impacto Izod}

Os ensaios de resistência ao impacto foram realizados de acordo com a norma ASTM D256 [16] em máquina tipo pêndulo CEAST 9050. Os corpos de prova foram obtidos através de moldagem por injeção, entalhados e submetidos ao teste de impacto do tipo Izod, empregando-se martelo com energia máxima de $0,5 \mathrm{~J}$ e temperatura ambiente de aproximadamente $21^{\circ} \mathrm{C}$. $\mathrm{O}$ valor de resistência ao impacto foi calculado através da média aritmética de dez corpos de prova para cada experimento. As amostras foram analisadas após condicionamento a uma temperatura média de $21^{\circ} \mathrm{C}$ e umidade relativa do ar em torno de $50 \%$ durante 48 horas.

\subsection{Avaliação da morfologia}

A morfologia das misturas PP/PP-g-MA/TPS foi avaliada em um microscópio eletrônico de varredura HITACH, modelo TM3000, utilizando-se uma voltagem de aceleração de $20 \mathrm{kV}$. Os corpos de prova das amostras obtidos por moldagem por injeção foram imersos em nitrogênio líquido para fratura criogênica. A superfície das amostras foi recoberta por uma fina camada de ouro em um metalizador.

\subsection{Análise termogravimétrica (TGA)}

A estabilidade térmica do polipropileno e das misturas PP/PP-g-MA/TPS foi avaliada em um analisador termogravimétrico T.A. Instruments, modelo Q500. Cerca de $15 \mathrm{mg}$ de cada amostra foi analisada. As análises foram efetuadas em atmosfera de nitrogênio, em faixa de temperatura de 30 a $700^{\circ} \mathrm{C}$ e taxa de aquecimento de $10^{\circ} \mathrm{C} / \mathrm{min}$.

\section{RESULTADOS E DISCUSSÃO}

\section{1 Índice de fluidez das misturas PP/PP-g-MA/TPS}

O índice de fluidez (MFI) foi utilizado para se obter uma estimativa das características reológicas do polímero fundido e com isso, determinar as condições de processamento. As medidas do índice de fluidez foram importantes na caracterização das misturas PP/PP-g-MA/TPS, de maneira a avaliar a influência do amido e do agente compatibilizante na processabilidade do polipropileno. A Tabela 2 apresenta os valores do índice de fluidez do PP virgem, do PP extrusado e das diferentes misturas processadas em extrusora de rosca dupla. $\mathrm{O}$ índice de fluidez do polipropileno extrusado sofreu um pequeno aumento, em comparação ao polipropileno virgem. Apesar disso, essa alteração no índice de fluidez foi muito pequena, demonstrando que o PP não sofre reações de degradação significativas durante o processamento na extrusora, nas condições experimentais adotadas. 
Tabela 2: Índice de fluidez do polipropileno virgem, do polipropileno extrusado e das misturas PP/PP-g-MA/TPS.

\begin{tabular}{l|l}
\hline COMPOSIÇÃo & MFI (g/10 min) \\
\hline PP virgem & $3,10 \pm 0,03$ \\
\hline PP extrusado & $3,31 \pm 0,06$ \\
\hline PP/TPS 80/20\% (m/m) & $3,95 \pm 0,17$ \\
\hline PP/PP-g-MA/TPS 77/3/20\% (m/m) & $4,14 \pm 0,14$ \\
\hline PP/PP-g-MA/TPS 75/5/20\% (m/m) & $4,69 \pm 0,06$ \\
\hline PP/PP-g-MA/TPS 73/7/20\% (m/m) & $5,22 \pm 0,12$ \\
\hline
\end{tabular}

Para a mistura PP/TPS 80/20 \% (m/m), a adição de $20 \%(\mathrm{~m} / \mathrm{m})$ do amido termoplástico proporcionou um pequeno aumento no índice de fluidez do polipropileno (Tabela 2). Para as misturas processadas em presença do agente compatibilizante (PP-g-MA) um aumento no índice de fluidez dessas misturas com o aumento do teor de PP-g-MA presente na mistura foi observado. Esse aumento pode ser explicado pelo valor do índice de fluidez do compatibilizante, que segundo o fabricante é de $109 \mathrm{~g} / 10 \mathrm{~min}\left(190^{\circ} \mathrm{C} / 2,16 \mathrm{~kg}\right)$. Logo, a adição de uma pequena quantidade desse material nas misturas PP/TPS promoveu o aumento nos valores do índice de fluidez observado. Os resultados obtidos mostraram que a adição conjunta do amido e do agente compatibilizante contribui para uma melhor processabilidade do polímero sintético. Esse fato é importante, uma vez que a melhor processabilidade das misturas pode contribuir para uma melhor dispersão da fase de amido termoplástico na matriz de polipropileno.

FERREIRA et al., [7] avaliaram o efeito da adição de argila organofílica em misturas de amido termoplástico e polipropileno (TPS/PP 70:30\% m/m), processadas em extrusora de rosca dupla. O teor de argila presente nos compósitos foi de 1,0, 2,5 e 5,0 \%, em relação a massa total da mistura. O polipropileno graftizado com anidrido maleico (PP-g-MA), Polybond 3200, foi adicionado às misturas como agente compatibilizante. As características de fluidez dos diferentes materiais obtidos foram investigadas através de reometria capilar e os valores de viscosidade foram reportados como viscosidade aparente. Segundo os autores, a mistura processada em presença do agente compatibilizante foi a que apresentou os menores valores de viscosidade, sob a taxa de cisalhamento aplicada [7]. Semelhantemente ao observado no presente trabalho, a adição do agente compatibilizante diminui a viscosidade da mistura e melhora a processabilidade do material. A diminuição da viscosidade da mistura final contribui para a quebra da fase dispersa em gotas menores e, consequentemente, melhora a dispersão da fase de amido pela matriz de PP.

\subsection{Morfologia das misturas PP/PP-g-MA/TPS}

Em misturas poliméricas, o estudo da morfologia do produto final é muito importante, já que muitas das propriedades do material, principalmente as propriedades mecânicas, dependem fortemente do tipo de morfologia apresentada pelas misturas [17]. Existem diversos fatores que são responsáveis pelo tipo de morfologia desenvolvida por uma mistura polimérica como: natureza dos componentes, razão de viscosidade de ambos os polímeros na temperatura de mistura, composição da mistura e condições de processamento [18]. Entre todos os fatores que afetam a formação da morfologia, a adesão interfacial entre os componentes da mistura apresenta um papel relevante nas propriedades mecânicas das misturas poliméricas. Através da técnica de microscopia eletrônica de varredura (MEV) foi possível a análise da morfologia e da interação entre as fases de polipropileno e amido termoplástico nas misturas PP/PP-g-MA/TPS. As micrografias obtidas também permitiram avaliar a dispersão da fase de amido pela matriz de PP, com o aumento do teor do agente compatibilizante PP-g-MA. A Figura 1 mostra as micrografias obtidas por MEV para a mistura PP/TPS 80/20\% $(\mathrm{m} / \mathrm{m})$ processada sem o agente compatibilizante, com aumentos de 500 e 1000 vezes. 

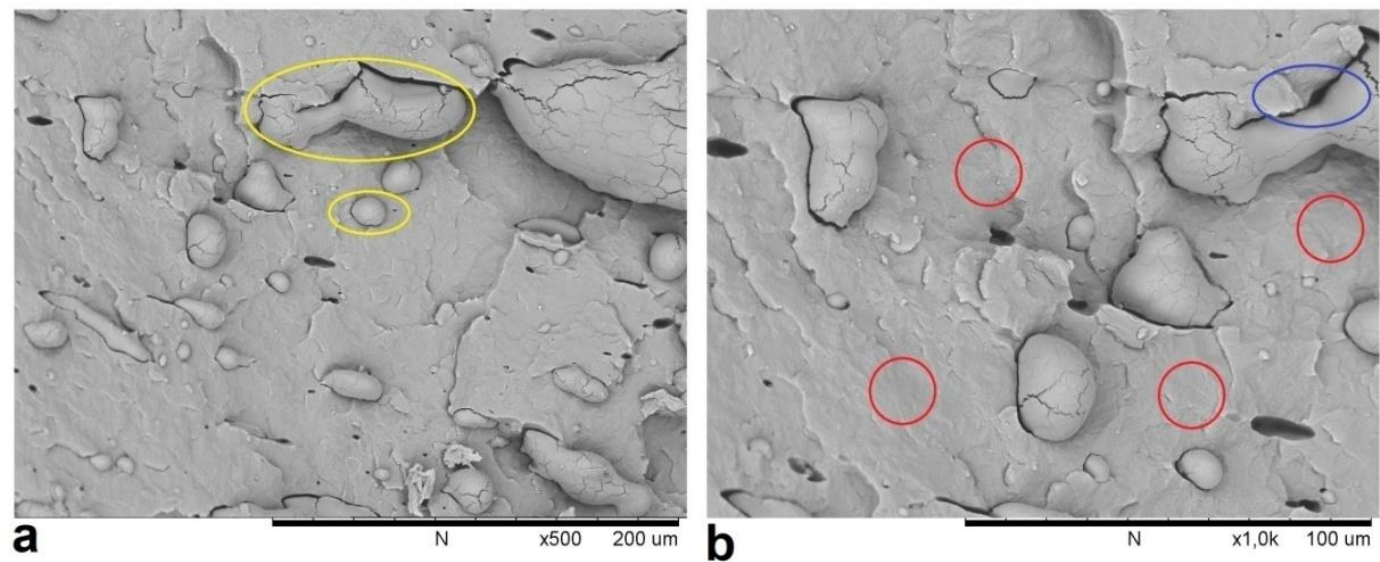

Figura 1: Micrografias obtidas por MEV da mistura PP/TPS 80/20\% (m/m) com diferentes aumentos: (a) 500 vezes e (b) 1000 vezes.

As micrografias da mistura mostram uma distribuição heterogênea da fase de amido termoplástico pela matriz de PP, sendo possível observar na superfície fraturada da amostra vários pontos onde há ausência da fase do polissacarídeo. Esses pontos onde há ausência da fase de amido são destacados na micrografia da Figura 1b por círculos vermelhos. Comportamento semelhante foi observado por OLIVEIRA et al., [19] na avaliação morfológica de misturas de polipropileno e amido termoplástico. Também é possível notar na micrografia da Figura 1a que a fase de amido termoplástico está mal dispersa pela matriz de PP, o que pode ser confirmado pela observação de gotas de amido de diferentes formatos e tamanhos. Duas gotas de amido de diferentes tamanhos são destacadas na micrografia da Figura 1a por elipses amarelas.

Esses resultados mostram que para essa mistura, as condições de processamento na extrusora não levaram a quebra de maneira eficiente da fase de amido e não possibilitaram uma dispersão eficiente do polissacarídeo na matriz de polipropileno. Essa mistura mostra características típicas as de um compósito imiscível, devido à falta de interação interfacial entre as fases de polipropileno e TPS. A micrografia obtida com 1000 vezes de aumento (Figura 1b) mostra a presença de espaços vazios entre as duas fases. Na micrografia da Figura 1b um espaço vazio entre as duas fases é destacado na figura através de uma elipse de cor azul.

A Figura 2 apresenta as micrografias das misturas PP/PP-g-MA/TPS obtidas com diferentes teores do agente compatibilizante. 
a

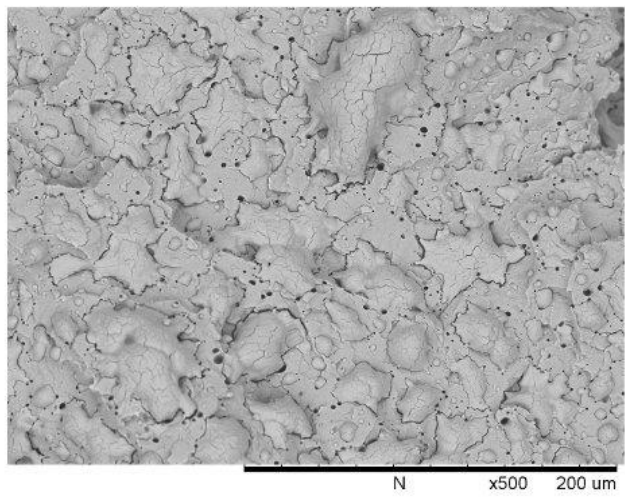

b

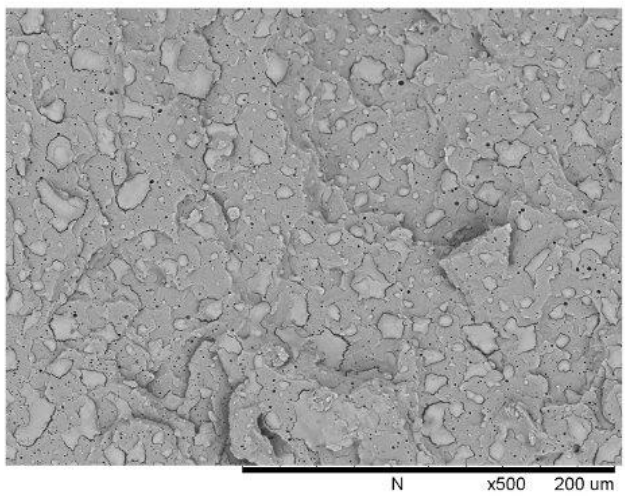

C

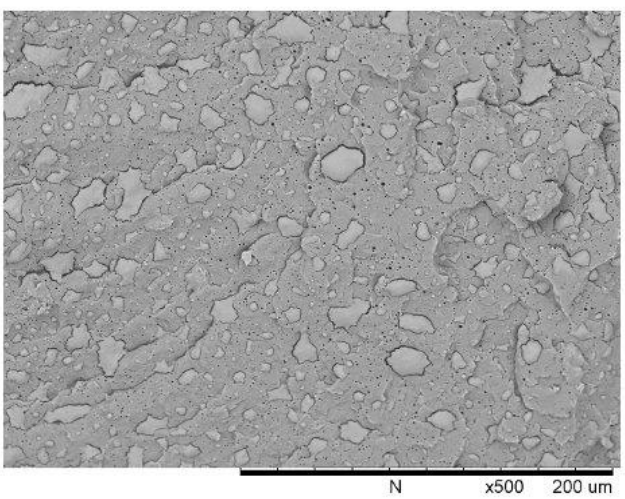

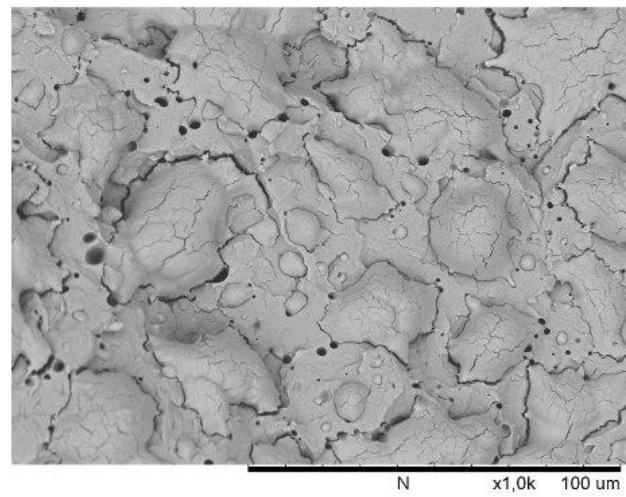
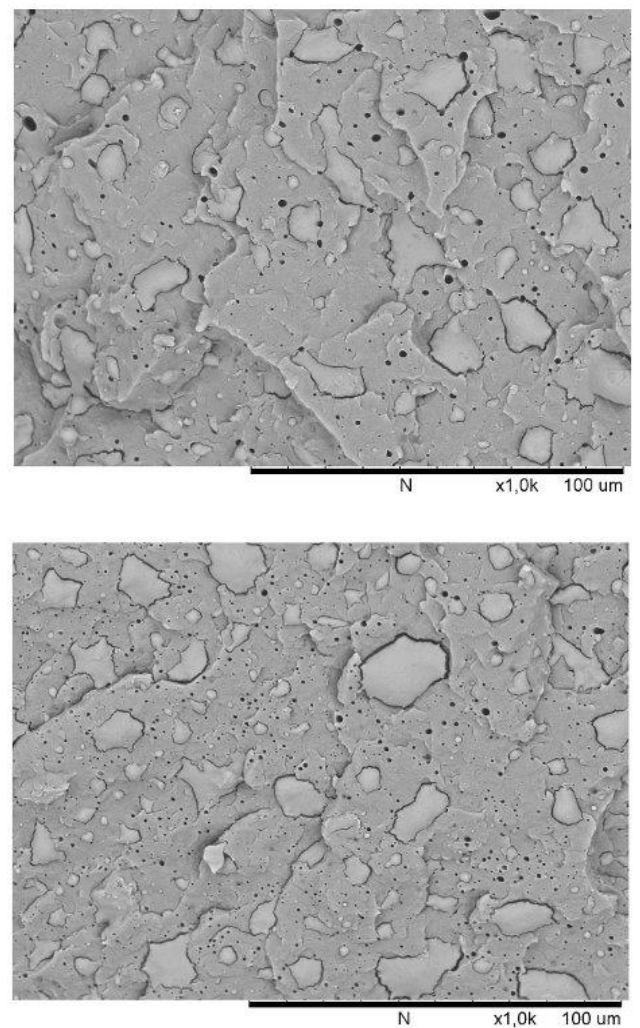

Figura 2: Micrografias obtidas por MEV das misturas PP/PP-g-MA/TPS: (a) PP/PP-g-MA/TPS 77:3:20, (b) PP/PP-gMA/TPS 75:5:20, (c) PP/PP-g-MA/TPS 73:7:20.

As micrografias dessas misturas mostram uma distribuição mais homogênea da fase de amido na matriz de polipropileno, sendo possível observar que a fase de amido está bem mais distribuída na matriz de PP (Figuras 2a, 2b e 2c). Também é possível observar através das micrografias que a fase de amido foi quebrada em gotas menores. O diâmetro dessas gotas de amido parece diminuir com o aumento do teor de agente compatibilizante adicionado à mistura PP/TPS. Isso mostra que, na presença do agente compatibilizante, as condições de processamento na extrusora de rosca dupla foram suficientes para quebrar a fase de amido em gotas de menor diâmetro e diminuir a possibilidade de ocorrência de coalescência da fase dispersa.

KONING et al., [20] afirmam em seu trabalho de revisão sobre estratégias para a compatibilização de misturas poliméricas, que a presença de moléculas do agente compatibilizante na superfície das partículas da fase dispersa, impede que ocorra a coalescência da fase dispersa durante o processamento das misturas. Nessas misturas, os agentes compatibilizantes são capazes de gerar e estabilizar uma morfologia mais fina. A emulsificação de misturas poliméricas tem sido proposta como o método mais eficiente para a obtenção de uma morfologia de fase fina e, consequentemente, melhores propriedades mecânicas do material final. A adição do agente compatibilizante PP-g-MA nas misturas PP/TPS promoveu a obtenção de materiais com dispersão mais eficiente do amido termoplástico no polipropileno e que apresentam morfologia mais fina e 
melhores propriedades de adesão devido a diminuição da tensão interfacial, o que pode promover a obtenção de propriedades mecânicas superiores.

CERCLÉ et al. [4] avaliaram a eficiência de compatibilização de copolímeros de polietileno graftizado com anidrido maleico em misturas de PE/TPS 80:20\% (m/m). Os copolímeros foram adicionados à mistura em concentrações de até $20 \%(\mathrm{~m} / \mathrm{m})$. A observação da superfície fraturada das misturas por microscopia eletrônica de varredura (MEV) mostrou que a adição do agente compatibilizante nas misturas proporcionou uma redução significativa no diâmetro da fase dispersa de amido. A mistura processada com $20 \%(\mathrm{~m} / \mathrm{m}) \mathrm{do}$ copolímero graftizado mostra um diâmetro médio da fase dispersa de $600 \mathrm{~nm}$ [4].

Em um trabalho publicado recentemente na literatura [21], os autores avaliaram a morfologia de misturas de polietileno de baixa densidade e polietileno linear de baixa densidade com amido termoplástico (LDPE/LLDPE/TPS). As misturas LDPE/LLDPE 70:30\% $(\mathrm{m} / \mathrm{m})$ foram processadas em extrusora de rosca dupla com 5-20 \% (m/m) de TPS e $3 \%(\mathrm{~m} / \mathrm{m})$ de polietileno graftizado com anidrido maleico (PE-g-MA). Os resultados obtidos por microscopia eletrônica de varredura mostraram uma boa dispersão das partículas de amido pelas matrizes de polietileno. Segundo os autores, a boa dispersão da fase de amido pela matriz é relacionada à presença de uma interação química do anidrido maleico (PE-g-MA) com os grupos hidroxila presentes no amido. A presença dessa interação possibilita a criação de uma interface nas misturas e diminui a tensão interfacial entre as fases.

\subsection{Propriedades mecânicas de tração}

As propriedades mecânicas do polipropileno e das misturas PP/PP-g-MA/TPS foram investigadas através de testes sob tração. As curvas de tensão-deformação das amostras de PP e de suas misturas com o amido termoplástico são apresentadas na Figura 3. Através da observação das curvas é possível verificar que a adição do amido termoplástico no polipropileno causa uma redução significativa na deformação na ruptura desse polímero. A resistência à tração do PP sofre uma ligeira queda com a adição do polissacarídeo.

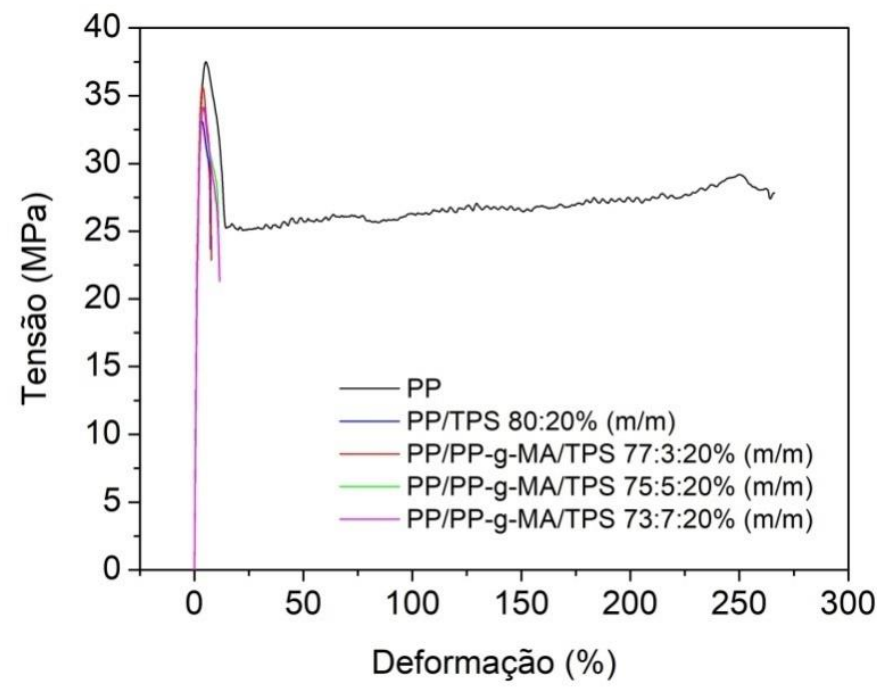

Figura 3: Curvas de tensão-deformação do polipropileno e das misturas PP/PP-g-MA/TPS.

Os valores das propriedades mecânicas, obtidas a partir das curvas de tensão-deformação: módulo de elasticidade (E), tensão máxima $\left(\sigma_{\max }\right)$ e deformação na ruptura $\left(\varepsilon_{\max }\right)$ são apresentados na Tabela 3. 
Tabela 3: Propriedades mecânicas do polipropileno e das misturas PP/PP-g-MA/TPS.

\begin{tabular}{l|l|l|l}
\hline COMPOSıÇÃo & $\begin{array}{l}\text { MÓDULO DE } \\
\text { YOUNG (MPa) }\end{array}$ & $\begin{array}{l}\text { TENSÃO MÁXIMA } \\
\text { (MPa) }\end{array}$ & $\begin{array}{l}\text { DEFORMAÇÃO NA } \\
\text { RUPTURA (\%) }\end{array}$ \\
\hline PP & $1407,67 \pm 32,33$ & $36,26 \pm 0,71$ & $271,49 \pm 44,10$ \\
\hline PP/TPS 80/20\% (m/m) & $1469,47 \pm 23,17$ & $32,77 \pm 0,41$ & $7,41 \pm 0,77$ \\
\hline PP/PP-g-MA/TPS 77/3/20\% (m/m) & $1506,47 \pm 11,85$ & $35,50 \pm 0,28$ & $7,74 \pm 1,14$ \\
\hline PP/PP-g-MA/TPS 75/5/20\% (m/m) & $1457,30 \pm 19,45$ & $34,52 \pm 0,32$ & $11,27 \pm 1,42$ \\
\hline PP/PP-g-MA/TPS 73/7/20\% (m/m) & $1415,35 \pm 29,49$ & $34,38 \pm 0,39$ & $11,57 \pm 1,56$ \\
\hline
\end{tabular}

O módulo de Young está associado à rigidez do material. Através da avaliação dos resultados do módulo de elasticidade apresentados na Tabela 3 é possível observar que a adição do amido termoplástico ao polipropileno resultou em um aumento de apenas $5 \%$ do módulo elástico. Esse resultado pode ser atribuido ao baixo teor de amido adicionado a matriz de PP. Alterações mais significativas nos valores de módulo em misturas de poliolefinas com TPS, geralmente são observados quando teores de amido acima de $30 \%(\mathrm{~m} / \mathrm{m})$ estão presentes na mistura [8]. PEDROSO e ROSA [22] avaliaram o efeito da adição do agente compatibilizante PE-g-GMA nas propriedades mecânicas em tração de misturas de amido com dois tipos de polietilenos de baixa densidade (virgem e reprocessado). As misturas foram processadas em extrusora de rosca dupla com e sem adição de $3 \%(\mathrm{~m} / \mathrm{m})$ do agente compatibilizante. O teor de amido presente nas misturas foi 30, 40 e $50 \%(\mathrm{~m} / \mathrm{m})$. Segundo os autores, a adição do PE-g-GMA às misturas PE virgem/amido provocou um aumento no módulo de Young apenas para as misturas obtidas com $50 \%$ (m/m) de amido [22]. Os resultados das propriedades mecânicas das misturas PP/PP-g-MA/TPS mostraram também que a adição de $3 \%$ do agente compatibilizante à mistura PP/TPS resultou em um aumento do módulo elástico, o que não foi observado para as misturas processadas com maiores teores do compatibilizante. Resultados referentes ao grau de cristalinidade devem ser obtidos de forma a elucidar esses resultados.

Com relação a resistência à tração das diferentes misturas PP/PP-g-MA/TPS obtidas em extrusora de rosca dupla, os resultados mostram que a adição de $3 \%(\mathrm{~m} / \mathrm{m})$ de PP-g-MA promoveu um aumento na resistência à tração da mistura em comparação com a mistura PP/TPS 80/20\%. O valor de resistência à tração para essa composição foi de 35,5 MPa, que é bem próximo ao valor apresentado pela amostra de PP. Esse resultado mostra que essa mistura com um teor de $20 \%$ de polímero biodegradável apresenta propriedades em tração superiores as apresentadas pelo polipropileno, se a deformação na ruptura não for um parâmetro de desempenho requerido. O aumento da concentração do agente compatibilizante, entretanto, não provocou alterações significativas dessa propriedade. Segundo PEDROSO e ROSA [22], esse aumento na resistência à tração pode ser atribuído a melhor interação entre as fases presentes na mistura. A avaliação das diferentes misturas através da microscopia eletrônica de varredura mostrou uma morfologia bem mais fina e estável com a adição do agente compatibilizante. OROMIEHIE et al. [8] avaliaram as propriedades mecânicas em tração de misturas de polietileno de baixa densidade e amido termoplástico, processadas em presença do agente compatibilizante LDPE-g-MA. Segundo os autores, a resistência à tração é inversamente proporcional ao teor de amido presente na mistura. A queda na resistência à tração ocorre devido a natureza frágil do amido termoplástico. Para as misturas processadas no presente trabalho, a queda da resistência à tração não foi tão acentuada devido ao baixo teor de amido termoplástico utilizado $(20 \% \mathrm{~m} / \mathrm{m})$.

Com relação a deformação na ruptura, os dados apresentados na Tabela 3 mostram uma queda significativa nos valores dessa propriedade para as misturas PP/PP-g-MA/TPS quando comparadas a deformação na ruptura do polipropileno. A deformação na ruptura diminui de $271 \%$ para $7 \%$ com a adição de $20 \%(\mathrm{~m} / \mathrm{m})$ de amido à matriz de PP. A adição do agente compatibilizante às misturas PP/TPS aumenta muito pouco a deformação na ruptura em comparação com a mistura PP/TPS 80/20 \% (m/m). De acordo com o trabalho de OROMIHIE et al. [8], em misturas de polietileno de baixa densidade (LDPE) e amido termoplástico (TPS), isso ocorre devido a dois principais fatores: (a) a incorporação física do amido na matriz de LDPE tornam mais fracas as forças de London entre as cadeias de LDPE e (b) o fato do amido, um polímero de baixo peso molecular, apresentar uma baixa deformação na ruptura quando comparado ao polietileno.

HUNEAULT e LI [23] avaliaram as propriedades mecânicas de misturas de amido termoplástico (TPS) e diferentes polímeros: polietileno de alta densidade, polipropileno, poliestireno, poli(ácido láctico) e policaprolactona. As misturas foram processadas em extrusora de rosca dupla com e sem a adição de diferentes copolímeros graftizados com anidrido maleico. No caso das misturas de TPS com PE e PP, o módulo de 
elasticidade e a resistência à tração apresentaram valores próximos aos valores dos polímeros puros, enquanto a deformação na ruptura foi drasticamente reduzida. Provavelmente, essa elevada redução na deformação na ruptura do polipropileno com a adição do amido termoplástico (TPS) possa ser atribuída a baixa deformação na ruptura do TPS em comparação ao polímero sintético.

\subsection{Resistência ao impacto Izod}

Medidas de resistência ao impacto Izod também foram utilizadas para a avaliação das propriedades mecânicas das misturas PP/PP-g-MA/TPS. Os ensaios foram realizados a temperatura ambiente $\left(23^{\circ} \mathrm{C}\right)$. A resistência ao impacto da mistura PP/TPS processada sem a adição do PP-g-MA e das misturas processadas em presença de 3 diferentes teores do agente compatibilizante são apresentados na Figura 4. A resistência ao impacto do polipropileno obtida foi de $23 \mathrm{~J} / \mathrm{m}$.

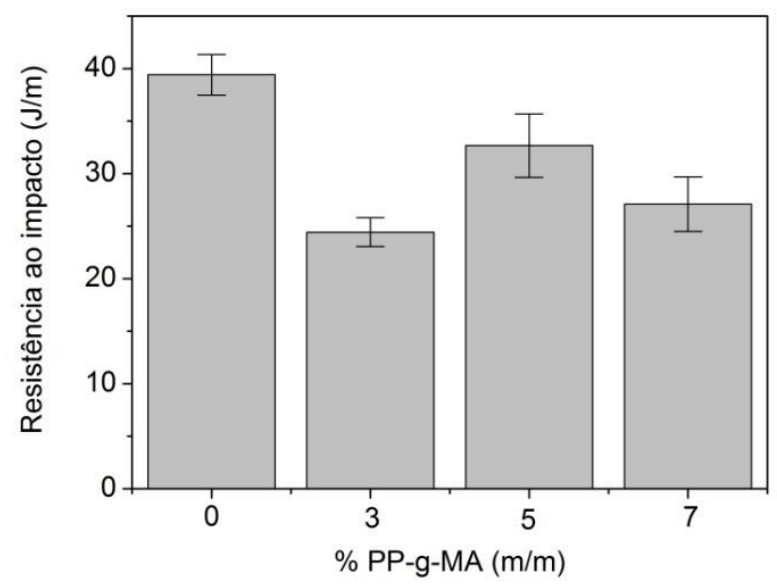

Figura 4: Resistência ao impacto Izod das misturas PP/PP-g-MA/TPS.

A adição de amido termoplástico ao polipropileno proporcionou um aumento significativo na resistência ao impacto do polímero sintético. Aumento semelhante foi observado por OLIVEIRA et al., [19] na avaliação da resistência ao impacto de misturas PP/TPS. A mistura PP/TPS 80:20 \% (m/m) mostrou uma resistência ao impacto de $39 \mathrm{~J} / \mathrm{m}$, o que corresponde a um aumento de $70 \%$ em relação a resistência ao impacto do PP. Para as misturas processadas em presença do PP-g-MA, a adição de $3 \%$ (m/m) do agente compatibilizante à mistura proporcionou uma redução significativa nessa propriedade. Com a adição de $5 \%$ $(\mathrm{m} / \mathrm{m})$ do PP-g-MA, a resistência ao impacto voltou a aumentar. Apesar disso, o valor encontrado para essa mistura ficou abaixo do valor obtido para a mistura processada sem a adição do agente compatibilizante. A adição de um maior teor do compatibilizante, $7 \%(\mathrm{~m} / \mathrm{m})$, não provocou alterações na resistência ao impacto em relação à adição de $5 \%$ do compatibilizante, levando-se em consideração o desvio-padrão.

São poucos trabalhos na literatura que avaliam as propriedades de resistência ao impacto de misturas de amido com polímeros sintéticos, como polietileno e polipropileno. TAGUET et al. [3], avaliaram a resistência ao impacto Izod de misturas de polietileno de alta densidade e amido termoplástico (HDPE/TPS) processadas com diferentes teores de glicerol como plastificante do amido. O agente compatibilizante PE-gMA foi adicionado ao HDPE sob diferentes concentrações. Os autores observaram um aumento significativo na resistência ao impacto da mistura com o aumento da concentração de agente compatibilizante presente na mistura [3].

TAGUET et al. [9], investigaram as propriedades mecânicas de impacto de misturas de polietileno de alta densidade (HDPE) e amido termoplástico (TPS) processadas sem e com a adição do agente compatibilizante (HDPE-g-MA). As misturas foram preparadas com $20 \%(\mathrm{~m} / \mathrm{m})$ de TPS e $80 \%(\mathrm{~m} / \mathrm{m}) \mathrm{de}$ HDPE. O amido termoplástico foi obtido com diferentes concentrações de glicerol como plastificante: 24,28 , 36 e $40 \%(\mathrm{~m} / \mathrm{m})$. Os resultados obtidos com a avaliação das diferentes misturas através de ensaios de resistência ao impacto mostraram que para as misturas processadas na presença do copolímero graftizado (HDPE-g-MA), os valores de resistência ao impacto foram mais baixos do que as misturas processadas sem a adição do agente compatibilizante. Segundo os autores, a compatibilização das misturas HDPE/TPS aumenta a área superficial das partículas de TPS devido a redução da fase dispersa de amido. Como o amido é um material mais frágil do que o polietileno, mesmo quando plastificado, as partículas de TPS atuam como 
concentradores de carga na matriz de polietileno, o que proporciona a falha do material através de propagação de trinca na interface matriz-fase dispersa. Quanto maior o número de partículas de TPS na matriz de PE, maior será a redução da resistência ao impacto [9].

Para as misturas PP/PP-g-MA/TPS avaliadas no presente trabalho, a redução da resistência ao impacto das misturas com a adição do agente compatiblizante pode ser explicada pelo aumento significativo da dispersão da fase de amido termoplástico na matriz de polipropileno. A adição do PP-g-MA proporcionou a quebra da fase de amido em gotas menores, como pode ser observado nas micrografias das misturas com diferentes concentrações do compatiblizante (Figura 2). Com a redução da fase dispersa de amido há um aumento da área superficial das partículas de TPS, o que leva a uma menor resistência ao impacto.

\subsection{Análise termogravimétrica}

No presente trabalho, o objetivo do uso da técnica de análise termogravimétrica (TGA) constituiu-se em determinar a temperatura de degradação das misturas PP/TPS processadas em presença do agente compatibilizante (PP-g-MA) e avaliar o efeito do PP-g-MA sobre a estabilidade térmica dos diferentes materiais obtidos. A Figura 5 mostra as curvas de análise térmica do polipropileno (PP), do amido termoplástico (TPS) e das diferentes misturas PP/PP-g-MA/TPS processadas em extrusora de rosca dupla. As correspondentes curvas de derivada de perda de massa dos diferentes materiais processados são apresentadas na Figura 6.

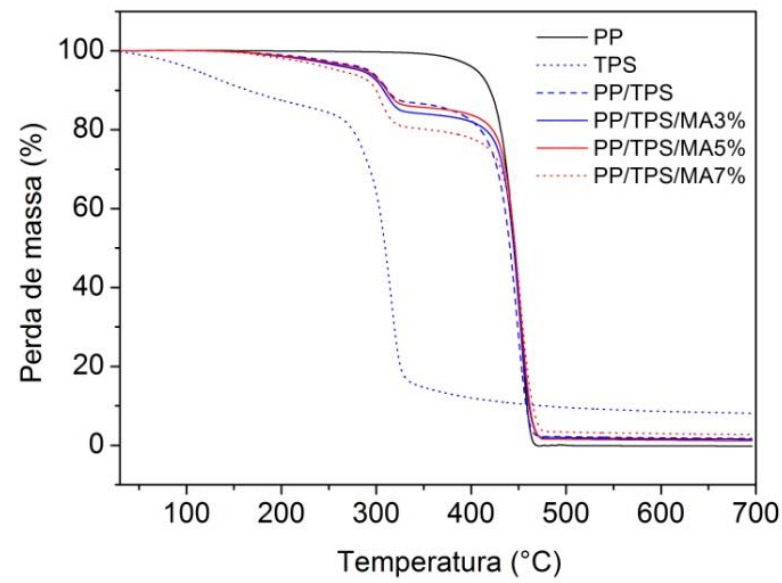

Figura 5: Curvas de análise térmica do polipropileno (PP), do amido termoplástico (TPS) e das misturas PP/PP-gMA/TPS

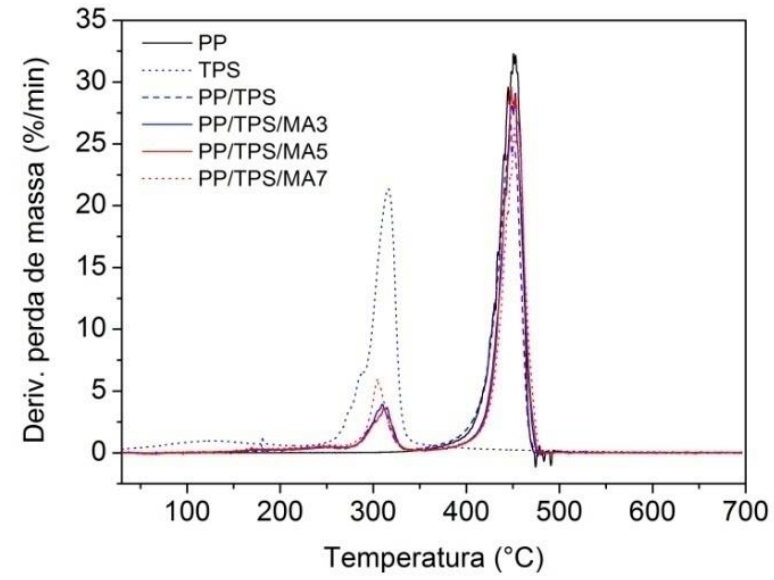

Figura 6: Curvas de derivada de perda de massa do polipropileno (PP), do amido termoplástico (TPS) e das misturas PP/PP-g-MA/TPS.

As curvas de análise térmica das misturas PP/TPS mostram a presença de três etapas de perda de massa. A primeira etapa de perda de massa tem início em torno de $160^{\circ} \mathrm{C}$ e é devido a evaporação do glicerol adicionado a fase de amido como plastificante. A segunda etapa de perda de massa é atribuída à decomposi- 
ção térmica da fase de amido e tem início em $290^{\circ} \mathrm{C}$. A degradação da fase de PP presente nas misturas tem início próximo de $430^{\circ} \mathrm{C}$ e se refere à terceira etapa de perda de massa observada nas curvas.

As temperaturas de degradação obtidas para o polipropileno e para as misturas PP/TPS processadas com e sem a adição do agente compatibilizante (PP-g-MA) são mostradas na Tabela 4. Os resultados obtidos com a avaliação das curvas de análise térmica mostraram que para as misturas PP/PP-g-MA/TPS, a presença do compatibilizante proporcionou um ligeiro aumento na estabilidade térmica dos produtos obtidos em comparação aos produtos derivados das misturas PS/TPS processadas sem adição do PP-g-MA. Os resultados foram evidenciados pela determinação da temperatura de início da degradação, $T_{3 o n s e t}$, e da temperatura de taxa máxima de degradação, $\mathrm{T}_{3 \mathrm{deg}}$, ambas referentes à terceira etapa de perda de massa, atribuída à degradação da fase de PP (Figuras 5 e 6 ).

Tabela 4: Temperatura de início da degradação, $T_{30 n s e t}$, e temperatura de taxa máxima de degradação, $T_{3 d e g}$, do polipropileno e das misturas PP/PP-g-MA/TPS.

\begin{tabular}{l|c|c}
\hline COMPOSIÇÃO & $\mathbf{T}_{\text {3onset }}\left({ }^{\circ} \mathbf{C}\right)$ & $\mathbf{T}_{\text {3deg }}\left({ }^{\circ} \mathbf{C}\right)$ \\
\hline PP & 429 & 451 \\
\hline PP/TPS 80/20\% (m/m) & 427 & 447 \\
\hline PP/PP-g-MA/TPS 77/3/20\% (m/m) & 434 & 453 \\
\hline PP/PP-g-MA/TPS 75/5/20\% (m/m) & 433 & 448 \\
\hline PP/PP-g-MA/TPS 73/7/20\% (m/m) & 435 & 452 \\
\hline
\end{tabular}

\section{CONCLUSÕES}

No presente trabalho a eficiência de compatibilização do copolímero graftizado PP-g-MA em misturas de polipropileno (PP) e amido termoplástico (TPS) foi avaliada. Resultados obtidos por microscopia eletrônica de varredura mostraram a obtenção de uma morfologia mais fina nas misturas PP/TPS com a incorporação do agente compatibilizante, além de uma maior interação interfacial entre os componentes da mistura. A avaliação das propriedades mecânicas das misturas mostrou que os materiais obtidos apresentam um grande potencial para utilização como substituintes do polipropileno em aplicações onde a deformação na ruptura não seja um parâmetro de desempenho mecânico significativo. Os diferentes materiais obtidos mostraram um ligeiro aumento na estabilidade térmica das misturas com o aumento do teor do agente compatibilizante.

\section{AGRADECIMENTOS}

O presente trabalho foi realizado com apoio da Coordenação de Aperfeiçoamento de Pessoal de Nível Superior - Brasil (CAPES) - Código de Financiamento 001. Os autores também agradecem ao Conselho Nacional de Desenvolvimento Científico e Tecnológico $(\mathrm{CNPq})$ e a Fundação Carlos Chagas Filho de Amparo à Pesquisa do Estado do Rio de Janeiro (FAPERJ) pelo apoio financeiro, a BRASKEM e a Ingredion Brasil Ingredientes Industriais Ltda. pela doação do polipropileno e do amido de milho, respectivamente.

\section{BIBLIOGRAFIA}

[1] PRACHAYAWARAKORN, J., SANGNITIDEJ, P., BOONPASITH, P., "Properties of thermoplastic rice starch composites reinforced by cotton fiber or low-density polyethylene", Carbohydrate Polymers, v. 81, pp. 425-433, Mar. 2010.

[2] SHAFIE SABET, S., KATBAB, A.A., "Interfacially compatibilized poly(lactic acid) and poly(lactic acid)/polycaprolactone/organoclay nanocomposites with improved biodegradability and barrier properties: effects of the compatibilizer structural parameters and feeding route", Journal of Applied Polymer Science, v. 111, pp. 1954-1963, 2009.

[3] TAGUET, A., HUNEAULT, M.A., FAVIS, B.D., "Interface/morphology relationships in polymer blends with thermoplastic starch", Polymer, v. 50, pp. 5733-5743, Sep. 2009.

[4] CERCLÉ, C., SARAZIN, P., FAVIS, B.D., "High performance polyethylene/thermoplastic starch blends through controlled emulsification phenomena”, Carbohydrate Polymers, v. 92, pp. 138-148, 2013.

[5] MAGALHÃES, N.F., ANDRADE, C.T., "Thermoplastic corn starch/clay hybrids: effect of clay type and content on physical properties", Carbohydrate Polymers, v. 75, pp. 712-718, 2009. 
[6] LI, H., HUNEAULT, M.A., "Comparison of sorbitol and glycerol as plasticizers for thermoplastic starch in TPS/PLA blends", Journal of Applied Polymer Science, v. 119, pp. 2439-2448, 2011.

[7] FERREIRA, W.H., KHALILI, R.R., FIGUEIRA JUNIOR, M.J.M., et al., "Effect of organoclay on blends of individually plasticized thermoplastic starch and polypropylene", Industrial Crops and Products, v. 52, pp. 38-45, 2014.

[8] OROMIEHIE, A.R., TAHERZADEH IARI, T., RABIEE, A. "Physical and thermal mechanical properties of corn starch/LDPE composites", Journal of Applied Polymer Science, v. 127, pp. 1128-1134, 2013.

[9] TAGUET, A., BUREAU, M.N., HUNEAULT, M.A., et al., "Toughening mechanisms in interfacially modified HDPE/thermoplastic starch blends", Carbohydrate Polymers, v. 114, pp. 222-229, 2014.

[10] KOROL, J., LENZA, J., FORMELA, K., "Manufacture and research of TPS/PE biocomposites properties", Composites: Part B, v. 68, pp. 310-316, 2015.

[11] AKRAMI, M., GHASEMI, I., AZIZI, H., et al., "A new approach in compatibilization of the poly(lactic acid)/thermoplastic starch (PLA/TPS) blends", Carbohydrate Polymers, v. 144, pp. 254-262, 2016.

[12] MARTINS, A.B., CATTELAN, A.K., SANTANA, R.M.C., "How the compatibility between polyethylene and thermoplastic starch can be improved by adding organic acids?", Polymer Bulletin, v. 74, pp. 1-16, Aug. 2017.

[13] ZHANG, C.L., FENG, L.F., GU, X.P., et al., "Efficiency of graft copolymers as compatibilizers for immiscible polymer blends", Polymer, v. 48, pp. 5940-5949, 2007.

[14] AMERICAN SOCIETY OF TESTING MATERIALS - ASTM, D1238: Standard Test Method for Melt Flow Rates of Thermoplastic by Extrusion Plastometer, New York, 2004.

[15] AMERICAN SOCIETY OF TESTING MATERIALS - ASTM, D638: Standard Test Method for Tensile Properties of Plastics, New York, 2004.

[16] AMERICAN SOCIETY OF TESTING MATERIALS - ASTM, D256: Standard Test Method for Determining the Izod Pendulum Impact Resistance of Plastics, New York, 2004.

[17] SAROJA, N., SHAMALA, T.L., THARANATHAN, R.N., "Biodegradation of starch-gpolyacrylonitrile, a packaging material, by Bacillus cereus", Process Biochemistry, v. 36, pp. 119-125, 2000.

[18] CHEN, L., GORDON, S.H., IMAM, S., "Starch graft poly(methyl accrylate) loose-fill foam: preparation, properties and degradation", Biomacromolecules, v. 5, pp. 238-244, 2004.

[19] OLIVEIRA, C.I.R., KUROBA, K.H., ALMEIDA, V.P., et al., "Propriedades mecânicas e morfologia de misturas de polipropileno e amido termoplástico", Acta Scientiae \& Technicae, v. 3, n. 2, pp. 19-24, Dec. 2015.

[20] KONING, C., VAN, M.D., PAGNOULlE, C., et al., "Strategies for compatibilization of polymer blends", Progress in Polymer Science, v. 23, pp. 707-757, 1998.

[21] SABETZADEH, M., BAGHERI, R., MASOOMI, M., "Morphology and rheological properties of compatibilized low-density polyethylene/linear low-density polyethylene/thermoplastic starch blends", Journal Applied Polymer Science, v. 134, pp. 44719-44727, 2017.

[22] PEDROSO, A.G., ROSA, D.S., "Effects of compatibilizer PE-g-GMA on the mechanical, thermal and morphological properties of virgin and reprocessed LDPE/corn starch blends", Polymers for Advanced Technologies, v. 16, pp. 310-317, 2005.

[23] HUNEAULT, M.A., LI, H., "Preparation and properties of extruded thermoplastic starch/polymer blends", Journal of Applied Polymer Science, v. 126, pp. 96-108, Mar. 2012.

\section{ORCID}

Carlos Ivan Ribeiro de Oliveira

Vinícius Pereira de Almeida

Marisa Cristina Guimarães Rocha

Joaquim Teixeira de Assis https://orcid.org/0000-0003-0325-3874

https://orcid.org/0000-0003-1183-8565

https://orcid.org/0000-0002-5155-3399

https://orcid.org/0000-0002-4027-6939 